\title{
THE EXACT PEANO DERIVATIVE $\left({ }^{1}\right)$
}

\section{BY \\ H. WILLIAM OLIVER}

1. Introduction. A real-valued function $f(x)$, defined for $a \leqq x \leqq b$, is said to have an $n$th Peano derivative at $x_{0}, n=1,2, \cdots$ (also called an $n$th derivative of de la Vallée-Poussin), if there exist numbers $f_{1}\left(x_{0}\right), f_{2}\left(x_{0}\right), \cdots$, $f_{n}\left(x_{0}\right)$ such that

$$
f\left(x_{0}+h\right)=f\left(x_{0}\right)+h f_{1}\left(x_{0}\right)+\cdots+\frac{h^{n}}{n !}\left[f_{n}\left(x_{0}\right)+\epsilon\left(x_{0}, h\right)\right]
$$

where $\epsilon\left(x_{0}, h\right) \rightarrow 0$ as $h \rightarrow 0$. If $f$ has an $n$th Peano derivative $f_{n}\left(x_{0}\right)$ at $x_{0}$, then it has also a $k$ th Peano derivative $f_{k}\left(x_{0}\right), k=1,2, \cdots, n-1$, and $f_{1}\left(x_{0}\right)$ $=f^{\prime}\left(x_{0}\right)$, the ordinary first derivative. If $f_{n}\left(x_{0}\right)$ exists for every $x_{0}$ in an interval, we call $f_{n}$ an exact Peano derivative. This paper will be concerned with exact Peano derivatives.

If $f$ has an ordinary $n$th derivative, $f^{(n)}\left(x_{0}\right)$, at $x_{0}$, then Taylor's theorem shows that $f_{n}\left(x_{0}\right)$ exists and equals $f^{(n)}\left(x_{0}\right)$. The converse is not true: $f_{n}\left(x_{0}\right)$ may exist without $f^{(n)}\left(x_{0}\right)$ existing. As an example, we cite the function $f$ defined by: $f(x)=x^{3} \sin x^{-1}$ for $x \neq 0, f(0)=0$. For this function, $f_{2}(0)=0$, but $f^{\prime \prime}(0)$ does not exist. Thus the $n$th Peano derivative is a true generalization of the ordinary $n$th derivative if $n>1$.

The Peano derivative has been studied particularly by Denjoy [2]( $\left.{ }^{2}\right)$, Marcinkiewicz and Zygmund [5], and Marcinkiewicz [4]. The latter two papers, so far as they relate to the Peano derivative, are concerned chiefly with the existence almost everywhere of this derivative. Denjoy studies the Peano derivative not only from this point of view, but also as an exact derivative. Denjoy's aims and methods differ from ours, but the results overlap to a certain extent, as described below.

The purpose of this note is to examine the properties of the exact Peano derivative (e.P.d.); in particular, to show that, in many respects, it resembles the ordinary derivative. If $g$ is an ordinary exact derivative, then $g$ possesses the following properties:

(i) $g$ is in the first class of Baire,

(ii) $g$ has the property of Darboux, i.e., takes on all intermediate values,

Presented to the Society, under the title Borel derivative and exact Peano derivatives, September 7, 1951; received by the editors April 17, 1953.

(1) The results presented in this paper were originally contained in a dissertation presented by me to the Faculty of the Division of Physical Sciences of the University of Chicago in candidacy for the degree of Doctor of Philosophy.

${ }^{(2)}$ Numbers in brackets refer to the bibliography at the end of the paper. 
(iii) $g$ satisfies a mean value theorem,

(iv) if $A>B$, the set $\{x ; A>g(x)>B\}$ is either void or of positive measure.

(Precise definitions are given in §2.) We shall show (Theorems 1, 2, and 6) that all these properties are also enjoyed by an exact Peano derivative. From these results, it follows (Theorems 3 and 4) that an e.P.d., which is bounded either above or below, is an ordinary derivative at all points; and that any e.P.d. is an ordinary derivative at "many" points, i.e., at all points of an everywhere dense, open set. This result is, in a certain sense, the best possible (Theorem 5). In particular, if $f_{n}(x)=0$ for all $x, f$ is a polynomial of degree $n-1$ at most.

Of these results, Denjoy has previously obtained the following: Theorem 1, Corollary to Theorem 3, and Theorem 4.

The author wishes to express here his sincere gratitude to Professor Antoni Zygmund for his inspiration and guidance in this work.

2. Definitions and preliminary results. We shall denote by $[a, b]$ and $(a, b)$, respectively, the closed and open intervals with end points $a<b$. An "interval" (unqualified) will denote ambiguously a closed or open or halfopen interval. We shall sometimes write $f_{0}(x)$ for $f(x)$. Throughout the paper $n$ will denote an arbitrary positive integer, unless otherwise specified.

We remark two facts about any Peano derivative, not necessarily exact. First, the Peano derivative is linear in the function whose derivative is taken, i.e., if $s$ and $t$ are constants, $(s f+t g)_{n}=s f_{n}+t g_{n}$, provided the two derivatives on the right exist. Second, we may rewrite (1) in the form:

$$
\frac{f(x+h)-f(x)-h f_{1}(x)-\cdots-\frac{h^{n-1}}{(n-1) !} f_{n-1}(x)}{h^{n} / n !}=f_{n}(x)+\epsilon(x, h),
$$

so that $f_{n}(x)$ is the limit, as $h \rightarrow 0$, of the quotient on the left.

Definition. A function $f(x)$, defined for $x \in[a, b]$, is said to have the property of Darboux (or to belong to $\mathcal{D}$ on $[a, b]$ ) if, on every subinterval $\left[a^{\prime}, b^{\prime}\right]$ of $[a, b], f$ takes on all intermediate values, i.e., if $f\left(a^{\prime}\right) \neq f\left(b^{\prime}\right)$ and if $C$ lies between $f\left(a^{\prime}\right)$ and $f\left(b^{\prime}\right)$, there is a value $c, a^{\prime}<c<b^{\prime}$, for which $f(c)=C$.

Darboux first showed that an exact first derivative enjoys this property, whence the name. We state without proof the following consequences of the Darboux property:

If $f$ is monotone or Darboux on $[a, b]$ and on $[b, c]$, then it is also on $[a, c]$.

If $f$ is monotone or Darboux on a neighborhood of each point of an interval, then it is also on the whole interval. $[a, b]$.

If $f$ is monotone on $(a, b)$ and Darboux on $[a, b]$, then it is monotone on 
If $f$ is monotone and Darboux on an interval, then it is continuous on that interval.

We shall use these properties in the sequel without explicit reference.

Definition. If $f$ has an nth Peano derivative at each point of an interval $[a, b]$, we say that $f$ satisfies the mean value theorems $\mathcal{X H}_{n}^{k}, k=0,1, \cdots, n-1$ (or that $f \in \mathcal{X}_{n}^{k}$ ), if, for each $x$ and $x+h \in[a, b]$, there is an $x^{\prime}$ between $x$ and $x+h$ such that:

$$
\frac{f_{k}(x+h)-f_{k}(x)-h f_{k+1}(x)-\cdots-\frac{h^{n-k-1}}{(n-k-1) !} f_{n-1}(x)}{h^{n-k} /(n-k) !}=f_{n}\left(x^{\prime}\right) .
$$

When $n=1$ and $k=0$, we have the ordinary mean value theorem for first derivatives. The mean value theorem for $n$th Peano derivatives referred to in $\S 1$, (iii) is this: if $f_{n}$ is an e.P.d., then $f \in \mathcal{X}_{n}^{k}, k=0,1, \cdots, n-1$.

The special case of $\mathcal{H}_{n}^{k}$ when the left side of (2) equals 0 , we refer to as Rolle's Theorem, $\mathcal{R}_{n}^{k}$. In the usual manner, $\mathcal{X}_{n}^{k}$ follows from $\mathbb{R}_{n}^{k}$ by adding a suitable polynomial to $f$. If $f$ has an $n$th e.P.d. on $[a, b]$ and if $y$ and $y+h$ are given in $[a, b]$, we set:

$$
g(x)=f(x)-\frac{f_{k}(y+h)-f_{k}(y)-\cdots-\frac{h^{n-k-1}}{(n-k-1) !} f_{n-1}(y)}{h^{n-k} /(n-k) !} \cdot \frac{(x-y)^{n}}{n !} .
$$

It follows immediately that:

$$
g_{k}(y+h)-g_{k}(y)-h g_{k+1}(y)-\cdots-\frac{h^{n-k-1}}{(n-k-1) !} g_{n-1}(y)=0,
$$

i.e., that $g$ satisfies the hypotheses of $\mathcal{R}_{n}^{k}$; and that:

$$
g_{n}(x)=f_{n}(x)-\frac{f_{k}(y+h)-f_{k}(y)-\cdots-\frac{h^{n-k-1}}{(n-k-1) !} f_{n-1}(y)}{h^{n-k} /(n-k) !} .
$$

Applying the conclusion of $R_{n}^{k}$ to $g$, i.e., replacing $x$ by $x^{\prime}$ and $g_{n}\left(x^{\prime}\right)$ by 0 , the conclusion of $\mathcal{X}_{n}^{k}$ follows for $f$.

It is also possible to deduce $\mathcal{R}_{n}^{k}, k=0,1, \cdots, n-2$, from $\mathbb{R}_{n}^{n-1}$ and $\mathcal{X}_{n-1}^{k}$, as follows: we may write

$$
\frac{f_{k}(x+h)-f_{k}(x)-\cdots-\frac{h^{n-k-2}}{(n-k-2) !} f_{n-2}(x)-\frac{h^{n-k-1}}{(n-k-1) !} f_{n-1}(x)}{h^{n-k} /(n-k) !}=0
$$


in the form

$$
\frac{\frac{f_{k}(x+h)-f_{k}(x)-\cdots-\frac{h^{n-k-2}}{(n-k-2) !} f_{n-2}(x)}{h^{n-k-1} /(n-k-1) !}-f_{n-1}(x)}{h /(n-k)}=0
$$

and replace the first ratio, using $\mathcal{P H}_{n-1}^{k}$, to obtain:

$$
f_{n-1}\left(x^{\prime \prime}\right)-f_{n-1}(x)=0, \quad \text { for some } x^{\prime \prime} \text { between } x \text { and } x+h \text {. }
$$

We use $R_{n}^{n-1}$ to deduce from this last equation the existence of $x^{\prime}$ between $x$ and $x^{\prime \prime}$ for which $f_{n}\left(x^{\prime}\right)=0$, the conclusion required by $\mathcal{R}_{n}^{k}$.

We shall later (Theorem 2) carry out a proof of the mean value theorems $\mathcal{X}_{n}^{k}$ by induction on $n$. Since the theorem holds for $n=1$, the remarks of the two preceding paragraphs show that the induction will be completed by deducing $\mathcal{R}_{n}^{n-1}$ from $\mathscr{X}_{n-1}^{k}$.

3. An important lemma. In this section, we deduce the fundamental result stated in the following lemma.

LEMMA 1. If $f_{n}$ exists and is positive for all $x \in[a, b]$, if $f \in \mathcal{M}_{n-1}^{0}$, and if $f_{n-1} \in \mathcal{D}$, then $f_{n-1}$ is strictly increasing and continuous on $[a, b]$.

Before proceeding with the proof of Lemma 1, we prove two subsidiary lemmas.

Lemma A. If the hypotheses of Lemma 1 are satisfied, then $f_{n-1}$ is strictly increasing on any interval on which it is upper semi-continuous (u.s.c.).

Proof of Lemma A. Let $\left[a^{\prime \prime}, b^{\prime \prime}\right]$ be an interval on which $f_{n-1}$ is u.s.c., and let $\left[a^{\prime}, b^{\prime}\right]$ be an arbitrary subinterval of $\left[a^{\prime \prime}, b^{\prime \prime}\right]$. Because of the u.s.c., $f_{n-1}$ attains its maximum at some point in $\left[a^{\prime}, b^{\prime}\right]$. Let us assume there is an $x, a^{\prime} \leqq x<b^{\prime}$, for which this maximum is attained. Since $f_{n}(x)>0$, there is an $h, x<x+h<b^{\prime}$, such that:

$$
\frac{f(x+h)-f(x)-\cdots-\frac{h^{n-2}}{(n-2) !} f_{n-2}(x)-\frac{h^{n-1}}{(n-1) !} f_{n-1}(x)}{h^{n} / n !}>0 .
$$

We rewrite the left side of this inequality as:

$$
\frac{\frac{f(x+h)-f(x)-\cdots-\frac{h^{n-2}}{(n-2) !} f_{n-2}(x)}{h^{n-1} /(n-1) !}-f_{n-1}(x)}{h / n}
$$


and use the fact that $f \in \mathcal{X}_{n-1}^{0}$ to replace the first ratio by $f_{n-1}\left(x^{\prime}\right)$, for some $x^{\prime}, x<x^{\prime}<x+h$. We thus obtain $f_{n-1}\left(x^{\prime}\right)>f_{n-1}(x)$, which contradicts the assumption that $f_{n-1}$ attained its maximum at $x$. Thus $f_{n-1}$ attains its maximum on $\left[a^{\prime}, b^{\prime}\right]$ only at $b^{\prime}$. Since $\left[a^{\prime}, b^{\prime}\right]$ was an arbitrary subinterval of $\left[a^{\prime \prime}, b^{\prime \prime}\right], f_{n-1}$ is strictly increasing on $\left[a^{\prime \prime}, b^{\prime \prime}\right]$.

Lemma B. Let $g(x)$ be defined for $x \in[a, b]$. Let $F$ be a closed subset of $[a, b]$ and let $g$, restricted to $F$, be u.s.c. on $F$. For each component interval $(c, d)$ of the open complement of $F$, let $g$ be nondecreasing and u.s.c. on the closure $[c, d]$. Then $g$ is u.s.c. on $[a, b]$.

Proof of Lemma B. If $x \notin F, g$ is u.s.c. at $x$ by hypothesis. If $x \in F$ and is isolated on the right (or left) from $F$, then $x$ is the left (or right) end point of a component interval of the complement of $F$, and so is u.s.c. on the right (or left). If $x \in F$ is a point of accumulation from the right of $F$, choose $\delta>0$ so that $g(y)<g(x)+\epsilon$, for all $y \in F$ such that $x \leqq y \leqq x+\delta$. Since $x$ is a point of accumulation from the right of $F, \delta$ may be chosen so that $x+\delta \in F$. Let $x^{\prime}$ be any point, $x<x^{\prime} \leqq x+\delta$. If $x^{\prime} \in F, g\left(x^{\prime}\right)<g(x)+\epsilon$ by the choice of $\delta$. If $x^{\prime} \notin F, x^{\prime}$ belongs to a component interval, say $\left(c^{\prime}, d^{\prime}\right)$, of the complement of $F$. Since $x+\delta$ and $d^{\prime}$ both belong to $F, d^{\prime} \leqq x+\delta$, so that $g\left(d^{\prime}\right)<g(x)+\epsilon$. But $g$ is nondecreasing on $\left[c^{\prime}, d^{\prime}\right]$, so that $g\left(x^{\prime}\right) \leqq g\left(d^{\prime}\right)<g(x)+\epsilon$. Thus $g$ is u.s.c. on the right at each right-hand accumulation point of $F$; and, similarly, $g$ is u.s.c. on the left at each left-hand accumulation point. Thus $g$ is u.s.c. at all points of $[a, b]$.

We now return to the proof of Lemma 1.

We define $\bar{\Delta}_{h}^{k} f(x)$, for $k=1,2, \cdots$, as follows:

$$
\begin{aligned}
& \bar{\Delta}_{h}^{1} f(x)=f(x+h)-f(x), \\
& \bar{\Delta}_{h}^{k} f(x)=\bar{\Delta}_{2 h}^{k-1} f(x)-2^{k-1} \bar{\Delta}_{h}^{k-1} f(x),
\end{aligned}
$$

It is known that $h^{-k} \bar{\Delta}_{h}^{k} f(x) \rightarrow f_{k}(x)$ as $h \rightarrow 0$, whenever $f_{k}(x)$ exists [5].

Since $f_{n}(x)>0$ for all $x$, we shall have, upon putting $k=n$ in (3) and dividing by $(2 h)^{n-1}$ :

$$
\frac{\bar{\Delta}_{2 h}^{n-1} f(x)}{(2 h)^{n-1}}-\frac{\bar{\Delta}_{h}^{n-1} f(x)}{h^{n-1}}=\frac{\bar{\Delta}_{h}^{n} f(x)}{h^{n}} \cdot \frac{h}{2^{n-1}}>0,
$$

provided that $0<h<\delta=\delta(x)$. Let us set:

$$
F_{k}(x)=\frac{\bar{\Delta}_{2}^{n-1} f(x)}{\left(2^{-k}\right)^{n-1}}, \quad \quad k=1,2, \cdots .
$$

Then $F_{k}(x) \rightarrow f_{n-1}(x)$ as $k \rightarrow \infty$, and, from (4), with $h=2^{-k-1}$, we have:

$$
F_{k}(x)>F_{k+1}(x), \quad \text { for } x \in[a, b) \text { and } k>k_{0}(x) \text {. }
$$


Note that $k_{0}$ depends on $x$, i.e., that the sequence is decreasing at each point, but is not necessarily a decreasing sequence. Following Saks [6], we shall call such a sequence "quasi-nonincreasing." Saks shows that the limit $g$ of a quasi-nonincreasing sequence of continuous functions has the following property:

(5) if $N$ is any closed set, there is an $\left(a^{\prime}, b^{\prime}\right)$ such that $\left(a^{\prime}, b^{\prime}\right) \cdot N \neq 0$ and $g$, restricted to $F$, is u.s.c. on $F$, where $F=$ the closure of $\left(a^{\prime}, b^{\prime}\right) \cdot N$.

Thus $f_{n-1}$, as the limit of $F_{k}$, has the property (5).

Now let $Y$ denote the set of points in a neighborhood of which $f_{n-1}$ is strictly increasing; and let $N=(a, b)-Y . Y$ is clearly open, and so $N$ closed, in $(a, b)$. If we show that $N$ is void, then $f_{n-1}$ is strictly increasing on $(a, b)$. Since, by hypothesis, $f_{n-1} \in \mathcal{D}$ on $[a, b], f_{n-1}$ is strictly increasing and continuous on $[a, b]$. Thus the lemma is proved when $N$ is shown to be void.

Assume therefore that $N$ is not void. Since $N$ is closed, we can, by (5), find an interval $\left(a^{\prime}, b^{\prime}\right)$ such that $f_{n-1}$, restricted to $F$, is u.s.c. on $F=$ closure of $\left(a^{\prime}, b^{\prime}\right) \cdot N$. Now on each component interval of $Y$, in particular on each component interval $(c, d)$ of $\left[a^{\prime}, b^{\prime}\right]-F, f_{n-1}$ is strictly increasing. Since $f_{n-1} \in \mathcal{D}, f_{n-1}$ is strictly increasing and continuous on each $[c, d]$. By Lemma $\mathrm{B}, f_{n-1}$ is u.s.c. on $\left[a^{\prime}, b^{\prime}\right]$, and, by Lemma A, strictly increasing on $\left[a^{\prime}, b^{\prime}\right]$. But this contradicts the assumption that $\left(a^{\prime}, b^{\prime}\right) \cdot N \neq 0$, and we must discard the assumption that $N \neq 0$. Thus the lemma is proved.

All the subsequent results, with the exception of Theorems 1 and 5, depend upon this lemma.

\section{Basic properties of the exact Peano derivative.}

THEOREM 1. If $f_{n}$ exists at every point of $[a, b]$, then $f_{n}$ belongs to the first class of Baire.

Proof. Since $f_{n}$ exists everywhere, the first derivative $f_{1}=f^{\prime}$ exists everywhere and so $f$ is continuous. We consider the $n$th difference $\Delta_{h}^{n}$, defined as follows:

$$
\begin{aligned}
\Delta_{h}^{n} f(x)=\sum_{i=0}^{n}(-1)^{n-i}\left(\begin{array}{c}
n \\
i
\end{array}\right) f(x+i h) \\
\left(\text { where }\left(\begin{array}{c}
n \\
i
\end{array}\right)\right. \text { is the binomial coefficient). }
\end{aligned}
$$

For fixed $h \neq 0, \Delta_{h}^{n} f(x) / h^{n}$ is continuous in $x$. If we use (1) to replace each term $f(x+i h)$ in the sum above, and then perform the summation, we find that

$$
\Delta_{h}^{n} f(x)=h^{n} f_{n}(x)+h^{n} \epsilon
$$

where $\epsilon \rightarrow 0$ as $h \rightarrow 0$. Letting $h \rightarrow 0$ through a sequence of values $h_{k}$, we see that $\Delta_{h_{k}}^{n} f(x) / h_{\mathbf{k}}^{n} \rightarrow f_{n}(x)$ as $k \rightarrow \infty$, i.e., that $f_{n}$ is in the first class of Baire. 
THEOREM 2. If $f_{n}$ exists at every point of $[a, b]$, then

(i) $f_{n}$ has the property of Darboux on $[a, b]$,

(ii) $f_{n}$ satisfies the mean value theorems $\mathcal{X}_{n}^{k}, k=0,1, \cdots, n-1$, on $[a, b]$.

Proof. We proceed by induction on $n$, proving (i) and (ii) simultaneously. For $n=1$, (i) and (ii) are well known. Assume then that the theorem holds for $1,2, \cdots, n-1$. We first prove (i) for the special case when $f_{n}$ is bounded above or below throughout $[a, b]$.

Case I. By changing the sign of $f$, if necessary, we may assume that $f_{n}$ is bounded below, say $f_{n}(x)>M$. The function $g(x)=f(x)-M x^{n} / n$ ! then has $g_{n}(x)>0$, and, by induction, satisfies the other hypotheses of Lemma 1 . Thus $g_{n-1}$, and so also $f_{n-1}$, are continuous.

Now let $\left[a^{\prime}, b^{\prime}\right]$ be any subinterval of $[a, b]$. If $f_{n}\left(a^{\prime}\right) \neq f_{n}\left(b^{\prime}\right)$, we may assume $f_{n}\left(a^{\prime}\right)>f_{n}\left(b^{\prime}\right)$. Let $C$ be any number, $f_{n}\left(a^{\prime}\right)>C>f_{n}\left(b^{\prime}\right)$; we may (by considering $f(x)-C x^{n} / n$ !) assume that $C=0$. To prove (i), we are to show that $f_{n}(c)=0$ for some $c \in\left(a^{\prime}, b^{\prime}\right)$.

Now $f_{n}\left(a^{\prime}\right)>0$, so that there is an $h>0$ such that

$$
\frac{f\left(a^{\prime}+h\right)-f\left(a^{\prime}\right)-\cdots-\frac{h^{n-1}}{(n-1) !} f_{n-1}\left(a^{\prime}\right)}{h^{n} / n !}>0 .
$$

We rewrite the left side of this inequality in the form

$$
\begin{aligned}
& f\left(a^{\prime}+h\right)-f\left(a^{\prime}\right)-\cdots-\frac{h^{n-2}}{(n-2) !} f_{n-2}\left(a^{\prime}\right) \\
& \frac{h^{n-1 /(n-1) !}-f_{n-1}\left(a^{\prime}\right)}{h / n}
\end{aligned}
$$

and use the fact that $f \in \mathcal{O H}_{n-1}^{0}$, by induction, to replace the first ratio in the numerator. Thus:

$$
\frac{f_{n-1}\left(x^{\prime}\right)-f_{n-1}\left(a^{\prime}\right)}{h / n}>0, \quad \text { for some } x^{\prime}, a^{\prime}<x^{\prime}<a^{\prime}+h .
$$

Since $h>0, f_{n-1}\left(x^{\prime}\right)>f_{n-1}\left(a^{\prime}\right)$, so that $f_{n-1}$ does not attain its maximum at $a^{\prime}$. Since $f_{n-1}\left(b^{\prime}\right)<f_{n-1}\left(a^{\prime}\right), f_{n-1}$ does not attain its maximum at $b^{\prime}$.

Now let $c, a^{\prime}<c<b^{\prime}$, be a point where $f_{n-1}$ attains its maximum; such a point exists since $f_{n-1}$ is continuous. Arguing as in the preceding paragraph, we find, for each $h$, positive or negative, an $x^{\prime}$ between $c$ and $c+h$ such that:

$$
\frac{f(c+h)-f(c)-\cdots-\frac{h^{n-1}}{(n-1) !} f_{n-1}(c)}{h^{n} / n !}=\frac{f_{n-1}\left(x^{\prime}\right)-f_{n-1}(c)}{h / n} .
$$


The numerator on the right is nonpositive for all $h \neq 0$ and the expression on the left tends to $f_{n}(c)$ as $h \rightarrow 0$. Considering first $h \rightarrow 0+$ and then $h \rightarrow 0-$, we see that $f_{n}(c)=0$. Thus (i) holds in Case I.

We now turn to the general case, where $f_{n}$ is unrestricted. We define the set $U \subset[a, b]$ as follows:

(6) $U$ is the set of points $x \in[a, b]$ such that for every right neighborhood of $x$ or for every left neighborhood of $x, f_{n}$ is unbounded both above and below.

Then $B=[a, b]-U$ is the set of those $x$ such that there is a right neighborhood and a left neighborhood in each of which $f_{n}$ is bounded above or below. $B$ is clearly open in $[a, b]$, so that $U$ is closed. Each point of $U$ is a point of discontinuity of $f_{n}$; since $f_{n}$ is in the first class of Baire, the points of continuity of $f_{n}$ are everywhere dense; and so $U$ can contain no interval. Thus $U$ is nowhere dense.

Let $\left[a^{\prime}, b^{\prime}\right]$ be an arbitrary subinterval of $[a, b]$. Suppose first that $U \cdot\left[a^{\prime}, b^{\prime}\right]$ is void. Then, if $x \in\left[a^{\prime}, b^{\prime}\right]$, there is an interval $[x, x+\delta]$ and an interval $[x-\delta, x]$ in each of which $f_{n}$ is bounded above or below. By Case I, $f_{n} \in \mathcal{D}$ on $[x, x+\delta]$ and on $[x-\delta, x]$; so also on $[x-\delta, x+\delta]$; and, finally, since $x$ is an arbitrary point of $\left[a^{\prime}, b^{\prime}\right]$, on $\left[a^{\prime}, b^{\prime}\right]$.

Consider now the case $U \cdot\left[a^{\prime}, b^{\prime}\right] \neq 0, U \cdot\left(a^{\prime}, b^{\prime}\right)=0$. The argument of the last paragraph shows that $f_{n} \in \mathcal{D}$ on $\left(a^{\prime}, b^{\prime}\right)$. If $f_{n}$ is bounded above or below to the right of $a^{\prime}$ and to the left of $b^{\prime}$, the same argument shows that $f_{n} \in \mathcal{D}$ on $\left[a^{\prime}, b^{\prime}\right]$. If $f_{n}$ is unbounded above and below on $\left(a^{\prime}, b^{\prime}\right)$, then, since $f_{n} \in \mathcal{D}$ on $\left(a^{\prime}, b^{\prime}\right)$, it takes on all values, and so, a fortiori, all values between $f_{n}\left(a^{\prime}\right)$ and $f_{n}\left(b^{\prime}\right)$.

If $U \cdot\left(a^{\prime}, b^{\prime}\right)$ contains an isolated point $x$ of $U$, there is a one-sided neighborhood of $x$, say $[x, x+\delta]$, such that $U \cdot(x, x+\delta)=0$ and $f_{n}$ is unbounded above and below on $(x, x+\delta)$. Then $f_{n}$ takes on all values on $(x, x+\delta)$ $\subset\left[a^{\prime}, b^{\prime}\right]$, and so all values between $f_{n}\left(a^{\prime}\right)$ and $f_{n}\left(b^{\prime}\right)$.

The last possibility is that $U \cdot\left(a^{\prime}, b^{\prime}\right) \neq 0$ and contains no isolated points. Then any portion of $U$ strictly interior to $\left(a^{\prime}, b^{\prime}\right)$ is perfect; let $P$ denote such a portion. Since $f_{n}$ is in the first class of Baire, there is a point $x \in P$ such that $f_{n}$, restricted to $P$, is continuous at $x$. We may assume that $f_{n}(x)=0$ and that $f_{n}$ is unbounded both above and below in every right neighborhood of $x$. For each positive integer $k$, let $\delta_{k}>0$ be such that $\left|f_{n}\left(x^{\prime}\right)\right|<1 / k$, if $x^{\prime} \in P$ and $x<x^{\prime}<x+\delta_{k}$. Let $x_{k}$ be a point of $\left(x, x+\delta_{k}\right)$ such that $f_{n}\left(x_{k}\right)>k$, and let $\left(c_{k}, d_{k}\right)$ be the interval contiguous to $P$ containing $x_{k}$; then $f_{n}\left(c_{k}\right)<1 / k$. By what has been already proved, $f_{n} \in \mathcal{D}$ on $\left[c_{k}, x_{k}\right]$ and so takes on all values between $1 / k$ and $k$. Since $k$ may be arbitrarily large, and since $f_{n}(x)=0, f_{n}$ takes on all non-negative values in every interval $[x, x+\delta]$. Similarly, $f_{n}$ takes on all nonpositive values, and so all values. Thus in all cases, $f_{n}$ takes on all values between $f_{n}\left(a^{\prime}\right)$ and $f_{n}\left(b^{\prime}\right)$. Since $\left[a^{\prime}, b^{\prime}\right]$ was an arbitrary subinterval of $[a, b], f_{n} \in \mathcal{D}$ on $[a, b]$, and (i) is proved. 
To prove (ii), the mean value theorems $\mathcal{X}_{n}^{k}, k=0,1, \cdots, n-1$, we refer to our remarks in $\$ 2$, which showed that this induction proof is completed by proving $R_{n}^{n-1}$, i.e., if $f_{n-1}(x+h)=f_{n-1}(x)$, there is an $x^{\prime}$ between $x$ and $x+h$ for which $f_{n}\left(x^{\prime}\right)=0$. We carry out this proof here.

We may assume $h>0$. If $f_{n}$ is positive on $[x, x+h]$, then, by Lemma 1 , $f_{n-1}$ is strictly increasing on $[x, x+h]$. But this is impossible, since $f_{n-1}(x+h)$ $=f_{n-1}(x)$. Similarly, $f_{n}$ cannot be negative on $[x, x+h]$. Thus $f_{n}$ must assume the value zero, or take on both positive and negative values. In the latter case, since $f_{n} \in \mathcal{D}, f_{n}$ also takes on the value zero. Thus $\mathbb{R}_{n}^{n-1}$ is proved, and the proof of Theorem 2 is complete.

5. The exact Peano derivative as an ordinary derivative. Lemma 1 shows that $f_{n-1}$ is continuous if $f_{n}>0$, and an easy application of Theorem 2 shows that this is true also if $f_{n}$ is bounded above or below $\left({ }^{3}\right)$. Our next theorem shows that in this situation much more can be said, namely, that $f_{n-1}$ has an ordinary first derivative equal to $f_{n}$ everywhere.

THEOREM 3. If $f_{n}(x)$ exists and is bounded above or below throughout $[a, b]$, then $f_{n}(x)=f^{(n)}(x)$, the ordinary nth derivative, at every point $x \in[a, b]$.

Proof. Let $x_{0}$ be an arbitrary point of $[a, b]$. We shall prove that, for $k=0,1, \cdots, n-1$ :

$$
\frac{f_{k}\left(x_{0}+h\right)-f_{k}\left(x_{0}\right)-h f_{k+1}\left(x_{0}\right)-\cdots-\frac{h^{n-k-1}}{(n-k-1) !} f_{n-1}\left(x_{0}\right)}{h^{n-k} /(n-k) !} \rightarrow f_{n}\left(x_{0}\right),
$$

as $h \rightarrow 0$. Thus $f_{n}\left(x_{0}\right)$ is the $(n-k)$ th Peano derivative, and $f_{k+1}\left(x_{0}\right)$ the ordinary first derivative, of $f_{k}, k=0,1, \cdots, n-1$.

For simplicity of notation we shall set $x_{0}=0$. By changing the sign of $f$, if necessary, and by adding a suitable polynomial to $f$, we may assume that $f(0)=f_{1}(0)=\cdots=f_{n-1}(0)=0, f_{n}(0)=A>0$, and $f_{n}(x)>0$ throughout $[a, b]$. We then have to prove that:

$$
\frac{f_{k}(h)}{h^{n-k}} \rightarrow \frac{A}{(n-k) !},
$$

as $h \rightarrow 0, k=0,1, \cdots, n-1$.

For $k=0$, this follows from the fact that $A$ is the $n$th Peano derivative of $f$ at 0 . We shall make the proof by induction on $k$.

First we use $\mathscr{H}_{n}^{n-1}$ to deduce that $h^{-1}\left[f_{n-1}(x+h)-f_{n-1}(x)\right]=f_{n}\left(x^{\prime}\right)>0$, so

(3) One might expect that it would be possible to deduce this last result $\left(f_{n-1}\right.$ is continuous if $f_{n}$ is bounded) as a corollary to Lemma 1 by adding a polynomial of degree $n$ to $f$. This change in $f$ would result in adding a linear function to $f_{n-1}$, and one would then have to show that the resulting $(n-1)$ th derivative had the property of Darboux, in order to apply Lemma 1 . It seems simpler to avoid this complication by deferring the discussion of this case until after the proof of Theorem 2 . 
that $f_{n-1}$ is increasing on $[a, b]$; and, since $f_{n-1}(0)=0, f_{n-1}(x)$ has the sign of $x$. Similarly, we use $\mathcal{X}_{n-1}^{n-2}$ and deduce that $h^{-1}\left[f_{n-2}(x+h)-f_{n-2}(x)\right]=f_{n-1}\left(x^{\prime}\right)$, so that $f_{n-2}$ is increasing on $[0, b]$ and decreasing on $[a, 0]$ and $f_{n-2}(x)>0$ if $x \neq 0$. By repeated applications of this argument, we find that $f_{n-k}(x)>0$ for $x \neq 0$, if $k$ is even; and $f_{n-k}(x)$ has the sign of $x$, if $k$ is odd. For either odd or even $k, f_{n-k}(x) / x^{k}>0$ for all $x \neq 0$.

Suppose we have shown that $f_{k}(h) / h^{n-k} \rightarrow A /(n-k)$ ! as $h \rightarrow 0$, for $k$ $=0,1, \cdots, m-1$. Using (2), with $n=m+1, k=0, x=h$, and $h=\alpha h$, for $0<|\alpha|<1$, we obtain:

$$
\begin{aligned}
& \frac{f(h+\alpha h)-f(h)-\cdots-\frac{(\alpha h)^{m}}{m !} f_{m}(h)}{(\alpha h)^{m+1}}=\frac{f_{m+1}\left(h^{\prime}\right)}{(m+1) !}, h^{\prime} \text { between } h \text { and } h+\alpha h, \\
& f(h+\alpha h)-f(h)-\cdots-\frac{(\alpha h)^{m}}{m !} f_{m}(h)
\end{aligned}
$$

$(\alpha h)^{n}$

$$
=\frac{f_{m+1}\left(h^{\prime}\right)}{\left(h^{\prime}\right)^{n-m-1}}\left(\frac{h^{\prime}}{h}\right)^{n-m-1} \frac{1}{(m+1) ! \alpha^{n-m-1}} .
$$

Now $h^{\prime} / h>0$; thus, in view of the last remark in the preceding paragraph, the sign of the right side of the last equation is that of $\alpha^{n-m-1}$. If $n-m-1$ is even, $\alpha^{n-m-1}>0$; if $n-m-1$ is odd, $\alpha^{n-m-1}$ has the sign of $\alpha$. Thus:

$$
\frac{f(h+\alpha h)-f(h)-\cdots-\frac{(\alpha h)^{m-1}}{(m-1) !} f_{m-1}(h)}{(\alpha h)^{n}} \gtrless \frac{f_{m}(h)}{m ! h^{n-m}} \cdot \frac{1}{\alpha^{n-m}} .
$$

If $n-m-1$ is even, $\alpha^{n-m}$ has the sign of $\alpha$, and the upper inequality holds in (7); thus multiplication by $\alpha^{n-m}$ results in:

$$
\begin{aligned}
& \frac{f(h+\alpha h)-f(h)-\cdots-\frac{(\alpha h)^{m-1}}{(m-1) !} f_{m-1}(h)}{\alpha^{m} h^{n}} \gtrless \frac{f_{m}(h)}{m ! h^{n-m}}, \\
& \text { according as } \alpha>0 \text { or } \alpha<0 \text {. }
\end{aligned}
$$

If, on the other hand, $n-m-1$ is odd, then $\alpha^{n-m}>0$, and multiplication of (7) by $\alpha^{n-m}$ does not change the inequality in (7). Thus (8) holds for all values of $m$.

We write the left side of (8), omitting the factor $1 / \alpha^{m}$, in the form:

$$
\frac{f(h+\alpha h)}{(h+\alpha h)^{n}} \cdot(1+\alpha)^{n}-\sum_{i=0}^{m-1} \frac{\alpha^{i}}{i !} \frac{f_{i}(h)}{h^{n-i}}
$$


and use our induction assumption to replace $f_{i}(h) / h^{n-i}$ by $A /(n-i) !+\epsilon_{i}$ and $f(h+\alpha h) /(h+\alpha h)^{n}$ by $A / n !+\epsilon^{\prime}$, where the $\epsilon^{\prime} \mathrm{s} \rightarrow 0$ with $h$. We thus obtain:

$$
\begin{aligned}
\left(A / n !+\epsilon^{\prime}\right)(1+\alpha)^{n}-\sum_{i=0}^{m-1} \frac{\alpha^{i}}{i !}\left(A /(n-i) !+\epsilon_{i}\right) & \\
=A & {\left[(1+\alpha)^{n} / n !-\sum_{i=0}^{m-1} \alpha^{i} / i !(n-i) !\right]+\left[\epsilon^{\prime}(1+\alpha)^{n}-\sum_{i=0}^{m-1} \alpha^{i} \epsilon_{i} / i !\right] } \\
=A \cdot P+Q, & \text { where } Q \rightarrow 0, \text { for fixed } \alpha, \text { as } h \rightarrow 0 .
\end{aligned}
$$

Thus (8) has become:

$$
A \cdot P / \alpha^{m}+Q / \alpha^{m} \gtrless f_{m}(h) / m ! h^{n-m}, \quad \text { according as } \alpha>0 \text { or } \alpha<0 .
$$

Now

$$
\begin{aligned}
P & =\frac{1}{n !}\left[\sum_{i=0}^{n}\left(\begin{array}{c}
n \\
i
\end{array}\right) \alpha^{i}-\sum_{i=0}^{m-1} \frac{n !}{i !(n-i) !} \alpha^{i}\right] \\
& =\frac{1}{n !} \sum_{i=m}^{n}\left(\begin{array}{c}
n \\
i
\end{array}\right) \alpha^{i},
\end{aligned}
$$

so that

$$
P / \alpha^{m} \rightarrow\left(\begin{array}{c}
n \\
m
\end{array}\right) / n !=\frac{1}{m !(n-m) !}
$$

as $\alpha \rightarrow 0$.

If $\epsilon>0$ is given, we choose and fix $\alpha$, positive and negative in turn, so small that $P / \alpha^{m}=1 / m !(n-m) !+\eta$, with $|\eta|<\epsilon / 2 A$. We then choose $\delta$ so that, for all $h, 0<|h|<\delta,|Q|<\left|\alpha^{m}\right| \epsilon / 2$. Then, using first $\alpha<0$ and then $\alpha>0$ in (9), we have:

$$
\frac{A}{m !(n-m) !}-\epsilon<\frac{f_{m}(h)}{m ! h^{n-m}}<\frac{A}{m !(n-m) !}+\epsilon
$$

for all $h, 0<|h|<\delta$, i.e., $f_{m}(h) / h^{n-m} \rightarrow A /(n-m)$ !.

Thus the induction is complete, and the theorem is proved.

Corollary. If $f_{n}(x)=0$ for all $x \in[a, b]$, then $f$ is a polynomial of degree $n-1$ at most. Thus a function is determined (up to a polynomial of degree n-1) by its nth Peano derivative given everywhere in an interval.

THEOREM 4. If $f_{n}(x)$ exists at every point of $[a, b]$, then there is an everywhere dense, open set $G \subset[a, b]$ at each point of which the ordinary nth derivative exists.

Proof. Let $G=[a, b]-U$, where $U$ is the closed, nowhere dense set defined by (6). If $x \in G, f_{n}$ is bounded above or below in a right, and in a left, neighborhood of $x$. Thus the right- and left-hand ordinary $n$th derivatives 
exist at $x$ and are each equal to $f_{n}(x)$, so that the ordinary two-sided derivative exists at $x$.

THEOREM 5. If an interval $[a, b]$ and an everywhere dense, open set $G \subset[a, b]$ and an integer $n \geqq 2$ are given, there is a function $f$ which has an nth Peano derivative at every point of $[a, b]$ and for which the set of points where the ordinary nth derivative exists is exactly $G$.

Proof. We denote by $P$ the closed, nowhere dense set $[a, b]-G$. Let $\left\{\left(a_{k}, b_{k}\right)\right\}$ denote the sequence of intervals contiguous to $P$. We may clearly assume that $P$ is nonvoid. We define:

$$
\begin{aligned}
& g(x)=x^{n+1}(1-x)^{n+1} \sin x^{-3 n} \sin (1-x)^{-3 n}, \quad 0<x<1, \\
& g(0)=g(1)=0 .
\end{aligned}
$$

Then $g^{(n)}(x)$ exists for $0<x<1$, and $g_{n}(0)=g_{n}(1)=0$. We note that $x^{n} g^{\prime}(x)$ is unbounded in every right neighborhood of 0 , and that $(1-x)^{n} g^{\prime}(x)$ is unbounded in every left neighborhood of $1 . g$ is bounded on $[0,1],|g| \leqq 1$.

We now define:

$$
\begin{array}{rr}
f(x)=\left(x-a_{k}\right)^{n}\left(b_{k}-x\right)^{n} g\left(\frac{x-a_{k}}{b_{k}-a_{k}}\right), & a_{k}<x<b_{k}, \\
f(x)=0, & x \in P .
\end{array}
$$

We shall show that $f$ satisfies the requirements of the theorem.

Clearly $f^{(n)}(x)$ exists if $x \notin P$.

At each left end point $a_{k}$ of an interval contiguous to $P$, the right-hand Peano derivative $f_{n}\left(a_{k}\right)=0$; similarly, the left-hand Peano derivative $f_{n}\left(b_{k}\right)$ $=0$ at a right end point $b_{k}$.

Suppose that $x \in P$ is a point of accumulation of $P$ from the right and that $x+h>x$. If $x+h \in P$, then $h^{-n}[f(x+h)-f(x)]=0$. If $x+h \in\left(a_{k}, b_{k}\right)$, so that $x+h=a_{k}+h^{\prime}$, with $0<h^{\prime}<h$ and $h^{\prime}<b_{k}-a_{k}$, then:

$$
\left|\frac{f(x+h)-f(x)}{h^{n}}\right|=\left|\frac{f\left(a_{k}+h^{\prime}\right)}{h^{n}}\right|=\frac{h^{\prime n}}{h^{n}}\left(b_{k}-a_{k}-h^{\prime}\right)^{n}|g| \leqq\left(b_{k}-a_{k}\right)^{n} .
$$

Since $x$ is a right-hand accumulation point of $P, k \rightarrow \infty$ and $b_{k}-a_{k} \rightarrow 0$ as $h \rightarrow 0+$, and so the right-hand $n$th Peano derivative exists and equals zero. Similarly, we show that the left-hand $n$th Peano derivative is zero at each left-hand accumulation point of $P$. Thus $f_{n}(x)=0$ for all $x \in P$, and so $f$ has an $n$th Peano derivative everywhere on $[a, b]$.

To show that $f^{(n)}(x)$ cannot exist if $x \in P$, it suffices to show that $f^{\prime}(x)$ is discontinuous at each point of $P$. Now, for $a_{k}<x<b_{k}, f^{\prime}(x)$ is the sum of two bounded terms and the term 


$$
\left(b_{k}-a_{k}\right)^{-1}\left(x-a_{k}\right)^{n}\left(b_{k}-x\right)^{n} g^{\prime}\left(\frac{x-a_{k}}{b_{k}-a_{k}}\right) .
$$

Since $x^{n} g^{\prime}(x)$ is unbounded to the right of $0, f^{\prime}(x)$ is unbounded to the right of each $a_{k}$. Similarly, $f^{\prime}(x)$ is unbounded to the left of each $b_{k}$. If $x \in P$, every neighborhood of $x$ contains an $a_{k}$ or a $b_{k}$. Thus $f^{\prime}$ is unbounded in every neighborhood of $x$ and so discontinuous at $x$. This completes the proof of Theorem 5.

6. Another property of exact Peano derivatives. It has been shown by Denjoy [3] and Clarkson [1] that an exact ordinary derivative has the property stated in $\$ 1$, (iv). We utilize this result and the consequences of Theorem 4 to show that an exact Peano derivative has the same property.

THEOREM 6. If $f_{n}$ exists at each point of $[a, b]$, then, for every pair of real numbers $A>B$, the set $E$ :

$$
E=\left\{x \in[a, b] ; A>f_{n}(x)>B\right\}
$$

is either void or has positive measure.

Proof. Let $U$ denote the set defined in (6), and let $G=[a, b]-U$; then $f^{(n)}(x)$ exists for $x \in G$. If $U$ is void, then $f^{(n)}$ exists everywhere and we may apply the known result to the ordinary derivative $f^{(n)}$ to prove the theorem.

If $U \neq 0$, then either $U$ has an isolated point or there is a point of $U$ at which $f_{n}$, restricted to $U$, is continuous. We showed in the proof of Theorem 2 that, in a neighborhood of a point of either of these types, $f_{n}$ takes on all values, even when $f_{n}$ is considered on $G$ alone. Thus, for every choice of $A$ and $B, E \cdot G \neq 0$. Let $(c, d)$ be a component interval of $G$ such that $E \cdot(c, d) \neq 0$. Applying the known result to the ordinary derivative $f^{(n)}$ on $(c, d)$, we deduce that $E \cdot(c, d)$ has positive measure. This completes the proof.

\section{BIBLIOGRAPHY}

1. J. A. Clarkson, A property of derivatives, Bull. Amer. Math. Soc. vol. 53 (1947) pp. 124125.

2. A. Denjoy, Sur l'intégration des coefficients différentiels d'ordre supérieur, Fund. Math. vol. 25 (1935) pp. 273-326.

3. - Sur une propriêté des fonctions dérivées exactes, L'Enseignement Mathématique vol. 18 (1916) pp. 320-328.

4. J. Marcinkiewicz, Sur les sêries de Fourier, Fund. Math. vol. 27 (1937) pp. 38-69.

5. J. Marcinkiewicz and A. Zygmund, On the differentiability of functions and summability of trigonometrical series, Fund. Math. vol. 26 (1936) pp. 1-43.

6. S. Saks, On the generalized derivatives, J. London Math. Soc. vol. 7 (1932) pp. 247-251.

University of Chicago,

Chicago, Ill.

Williams College,

Williamstown, Mass. 